\title{
Melanin in Fonsecaea pedrosoi: a trap for oxidative radicals
}

\author{
Marcel ML Cunha', Anderson J Franzen², Sergio H Seabra², Marcelo H Herbst³, Ney V Vugman4, Luana P Borba', \\ Wanderley de Souza' ${ }^{1}$ and Sonia Rozenta*1
}

\begin{abstract}
Background: The pathogenic fungus Fonsecaea pedrosoi constitutively produces the pigment melanin, an important virulence factor in fungi. Melanin is incorporated in the cell wall structure and provides chemical and physical protection for the fungus.

We evaluated the production of nitric oxide (NO) in macrophages, the oxidative burst and the inducible nitric oxide synthase (i-NOS) activity in interactions between activated murine macrophages and $F$. pedrosoi. Experiments were carried out with or without tricyclazole (TC) treatment, a selective inhibitor of the dihydroxynaphthalene (DHN)melanin biosynthesis pathway in F. pedrosoi. The paramagnetisms of melanin and the TC-melanin were analysed by electron spin resonance. The fungal growth responses to $\mathrm{H}_{2} \mathrm{O}_{2}$ and to $\mathrm{S}$-nitroso-N-acetylpenicillamine (SNAP), a nitric oxide donor, were also evaluated.

Results: Melanised F.pedrosoi cells were more resistant to both $\mathrm{H}_{2} \mathrm{O}_{2}$ and NO. Nitrite was not detected in the supernatant of macrophages incubated with melanised fungal cells. However, i-NOS expression was unaffected by the presence of either untreated control F. pedrosoi or TC-treated F. pedrosoi. In addition, the inhibition of the DHN-melanin pathway by TC improved the oxidative burst capability of the macrophages.
\end{abstract}

Conclusion: The NO-trapping ability of F. pedrosoi melanin is an important mechanism to escape the oxidative burst of macrophages.

\section{Background}

Fonsecaea pedrosoi is a soil-borne dimorphic fungus and the major etiological agent of chromoblastomycosis, a chronic disease that can affect immunocompetent hosts. F. pedrosoi is usually limited to skin tissue, most commonly on the lower limbs. Infection usually occurs after exposure to the fungus via contaminated soil, splinters or sharp farm equipment, and results in long-term inflammation, suppurative granulomatous dermatitis and fibrosis $[1,2]$. The affected patients are typically low-income workers that engage in agricultural or manual labour in tropical and subtropical countries. Rarely, F. pedrosoi can also cause phaeohyphomycosis, in immunosuppressed patients [3].

The management of diseases caused by F. pedrosoi continues to be challenging. Treatment depends on an early

\footnotetext{
* Correspondence: rozental@biof.ufrj.br

${ }^{1}$ Instituto de Biofísica Carlos Chagas Filho (IBCCF), Centro de Ciências da Saúde

(CCS), Universidade Federal do Rio de Janeiro (UFRJ), Rio de Janeiro, RJ, Brazil
}

diagnosis and the use of systemic antifungal agents and local therapies, including the surgical removal of lesions. The suggested drug interventions are expensive, involving high doses of itraconazole and/or terbinafine (200 to $400 \mathrm{mg}$ and 250 to $500 \mathrm{mg}$, respectively) daily for over one year. Even with treatment, relapses are common $[4,5]$.

F. pedrosoi constitutively produces melanin [6], a pigment that is an important virulence factor in several human pathogenic fungi due to its anti-oxidative, thermostable, anti-radioactive, paramagnetic and metal binding properties. Melanins are present in both prokaryotic and eukaryotic organisms. These ubiquitous dark compounds are formed by the oxidative polymerisation of phenolic or indolic compounds. Melanins have been extensively studied and characterised as negatively charged amorphous compounds with quinone groups, hydrophobic and insoluble in organic solvents $[7,8]$. Efforts to elucidate the structure of melanins are not yet conclusive due to limitations of the biochemical and bio- 
physical analytical methods available. Electron spin resonance (ESR) can characterise pigments, including melanin, and reveals that a typical melanin spectrum falls between 3300 and 3500 gauss [7-9].

Franzen et al. $[10,11]$ reported that $F$. pedrosoi constitutively synthesises melanin in organelles named melanosomes through the DHN-pathway. In F. pedrosoi, melanin confers structural integrity as a cell wall constituent and immune protection through antigen masking. F. pedrosoi melanin also has anti-phagocytic properties, and is overexpressed during infection [5]. Inside melanosomes, melanin plays a role in the intracellular storage and regulation of calcium and iron ions [11]. The anti-phagocytic properties of $F$. pedrosoi's melanin were described after interaction with murine macrophages with or without activation with lipopolysaccharide (LPS) and interferon-gamma (IFN- $\gamma$ ) $[12,13]$. In addition, conidia from $F$. pedrosoi cultures treated with $16 \mu \mathrm{g} / \mathrm{ml}$ of tricyclazole (TC), a DHN-melanin pathway inhibitor, showed a higher susceptibility to activated murine macrophages when compared to untreated fungus [12].

Macrophages are found in granulomas of chromoblastomycosis lesions and may participate in the antigen presentation and innate immune response against $F$. pedrosoi [14]. To contain the growth of pathogens, activated macrophages release oxygen and nitrogen reactive intermediates. NO released by the activated macrophages are fungicidal against Histoplasma capsulatum [15], Cryptococcus neoformans and Sporothrix schenkii [16,17]. The anti-oxidative properties of fungal melanins $[18,19]$, their paramagnetism as revealed by ESR, and the melanin-iron (a known magnetic or paramagnetic metal depending on its oxidation state) association in $F$. pedrosoi raised the hypothesis; the trapping of free radicals by fungal melanin during interactions between macrophages and fungi is a mechanism of oxidative buffering.

The aims of the present work were the following: (I) to characterise the melanin of $F$. pedrosoi by ESR; (II) to investigate the $\mathrm{NO}$ production of activated macrophages against $F$. pedrosoi conidia; (III) to detect i-NOS activity during macrophage interactions with fungi; (IV) to evaluate fungal growth after treatment with $\mathrm{NO}$ and $\mathrm{H}_{2} \mathrm{O}_{2}$; and (V) to compare these approaches in conidia with or without TC treatment.

\section{Results}

\section{ESR spectrometry and microwave power saturation of melanins}

The ESR spectra of the control-melanin and TC-melanin present strikingly similar signals with a peak of 3480 gauss (with respect to line width, line shape, and $g$ value of 2.0023) (Fig. 1A). Progressive microwave power saturation shows that the paramagnetic centres in these melanins do not saturate under the experimental conditions.

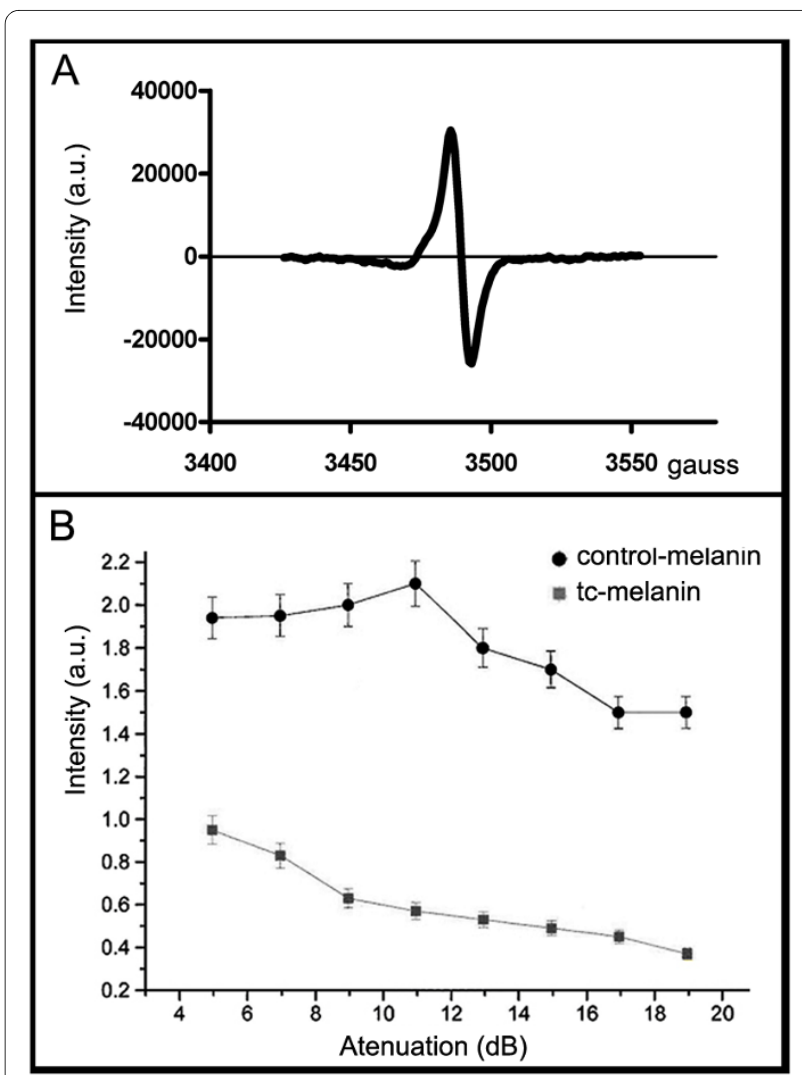

Figure 1 Electron spin resonance of melanins of $F$. pedrosoi. The ESR spectra (A) of control-melanin or TC-melanin present a single anisotropic line at $g=2.0023$. In (B), progressive microwave power saturation experiments show that the paramagnetic centres of these melanins do not saturate under the experimental conditions, and also that the control-melanin sample (black line, circles) has a higher spin relaxation rate than the TC-melanin sample (gray line, squares).

In addition, these experiments reveal that the controlmelanin has a higher spin relaxation rate than the TCmelanin (Fig. 1B). These observations suggest that the control-melanin is a more compact polymer than TCmelanin.

\section{Oxidative Burst}

The macrophage oxidative burst was analysed by the NBT assay. The activity of oxidative compounds released by activated macrophages was visualised through the precipitation of NBT-formazan (dark dye) around the fungus in all melanin-deficient systems. This precipitation occurs in response to superoxide molecules near the fungal cell wall (Fig. 2). Formazan precipitation was observed near S. cerevisiae (Fig. 2D) and F. pedrosoi grown in melanin-deficient conditions, such as with TC treatment (Fig. 2A) or low aeration (Fig. 2B). However, activity of the oxidative compounds was not detected in control F. pedrosoi conidia producing regular melanin (Fig. $2 \mathrm{C}$ ) or S. cerevi- 


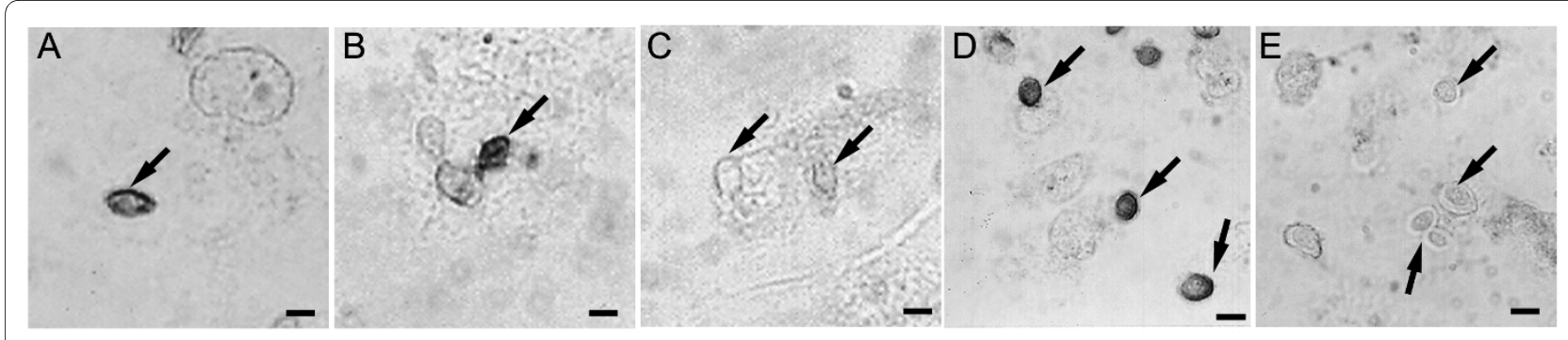

Figure 2 Light microscopy of the fungal interaction with activated murine macrophages. Light micrographs of activated murine macrophages after interaction in a 1:10 ratio with: (A) TC-treated F. pedrosoi conidia, (B) F. pedrosoi conidia grown under low aeration conditions, (C) control conidia of F. pedrosoi, (D) S. cerevisiae cells and (E) S. cerevisiae cells incubated with melanin from F. pedrosoi. Fungal cells are marked with arrows. The precipitation of NBT-formazan (dark dye) in response to the oxidative response was observed in A, B and D. Bars $=1 \mu \mathrm{m}$

siae supplemented with F. pedrosoi's control melanin (Fig. 2E).

\section{i-NOS expression revealed by immunofluorescence}

Immunocytochemistry studies with anti-i-NOS enzymes revealed that these enzymes were active in all models tested: macrophages alone (Fig. 3A, B); macrophages with control F. pedrosoi (Fig. 3C, D); or with TC-treated $F$. pedrosoi (Fig. 3E, F). Such data indicate that i-NOS expression was not inhibited in any tested condition.

\section{Nitrite evaluation}

After $24 \mathrm{~h}$ of interaction in cultures with F. pedrosoi and activated murine macrophages, the nitrite levels were reduced by $91 \%$ compared to the amount of nitrite observed in macrophage cultures without fungal interaction (Table 1). A similar reduction was observed when melanin extracted from control $F$. pedrosoi was added to a macrophage culture without fungal cells. Conidia isolated from TC-supplemented cultures yielded a detection of $81 \%$ more nitrite compared to non-infected macrophages after $24 \mathrm{~h}$ of interaction. Results obtained after 48 hours of interaction suggest a similar concentration endpoint of $\sim 50 \mu \mathrm{M}$ of nitrite for both macrophages alone or in interaction with TC-treated fungi.

\section{Fungal growth after direct activity of oxidative species}

The growth of TC-treated F. pedrosoi significantly decreased in comparison to the control after incubation with either $\mathrm{H}_{2} \mathrm{O}_{2}$ or SNAP $(\mathrm{P}<0.05$, Fig. 4). Differences were more prominent at concentrations of $0.005 \mathrm{M}$ of hydrogen peroxide and $0.3 \mathrm{M}$ of SNAP.

\section{Discussion}

Fungal melanins are a hot topic among mycologists and have been extensively characterised as virulence factors. Melanin pigments can protect pathogenic fungi from the mammalian host innate immune responses providing resistance: (I) to phagocytosis in C. neoformans, Paracoccidioides brasiliensis, S. schenkii and F. pedrosoi; (II) to killing by the host cell in the previously mentioned spe- cies as well as in Aspergillus fumigatus and Wangiella (Exophiala) dermatitidis; and (III) against oxidising agents in C. neoformans, Aspergillus spp. and S. schenkii $[8,20]$.

ESR characterizations of melanins correspond to a peak signal on the spectra near 3355 gauss. These data are coherent among several fungi regardless of the specific melanin biosynthetic pathway or even if the fungus is pathogenic, including C. neoformans [21]; Blastomyces dermatitidis [22], P. brasiliensis [23], H. capsulatum [24], S. schenckii [25] and W. dermatitidis [26], or not, as in the slime mould Fuligo septic [27], indicating that, at the molecular level, the structure of paramagnetic center is similar on these melanins.

The ESR characterisation of the samples revealed the presence of paramagnetic centres in both the controlmelanin and TC-melanin; however, the control-melanin sample was of a higher intensity indicating that the number of unpaired electrons (free radicals) was higher. Thus, these results indicate that the control-melanin is a polymer with more paramagnetic centres than the TC-melanin.

In the host environment, the expected iron species are diamagnetic $\mathrm{Fe}(\mathrm{II})$ and paramagnetic $\mathrm{Fe}(\mathrm{III})$. In our experiments $\mathrm{Fe}(\mathrm{III})$ was used as a nutrient since we used ferric ammonium citrate as the medium substrate. Fungal melanins are able to reduce $\mathrm{Fe}(\mathrm{III})$ to $\mathrm{Fe}(\mathrm{II})$, and this oxidative change prevents the formation of oxidative radicals when iron reacts with hydrogen peroxide, thus protecting the fungus from oxidative stress [28]. Cunha et al. [12] demonstrated that untreated F. pedrosoi has more abundant and homogeneous binding to cationised ferritin (a $\mathrm{Fe}(\mathrm{III})$ complex) on the cell wall surface than fungi treated with TC. At the time, the stronger binding was attributed to more anionic groups on the surface of the control and melanin's affinity to iron.

Experiments with melanin from C. neoformans [28] suggests that it acts as a redox buffer, changing its oxidative state according to the chemical stimuli in its environment. Thus, it is possible that melanin maximises its 


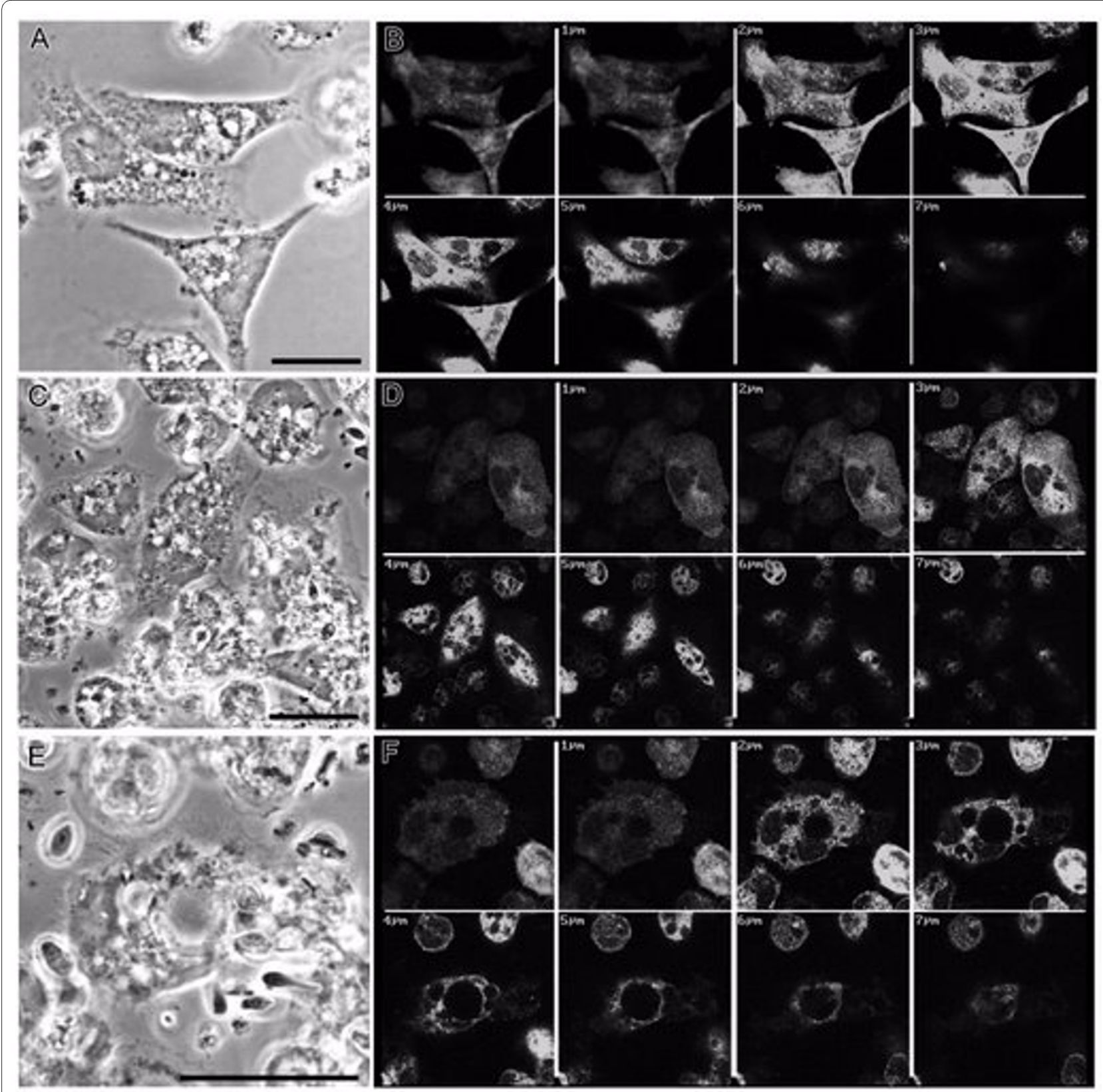

Figure $3 \boldsymbol{i}$-NOS expression upon fungus-macrophage interaction. Phase contrast microscopy ( $\mathrm{A}, \mathrm{C}$ and $\mathrm{E}$ ) and confocal immunocytochemistry ( $\mathrm{B}$, $D$ and $F$ ) images of activated murine macrophages alone (A-B), activated murine macrophages with untreated F. pedrosoi (C-D) or with TC-treated $F$. pedrosoi (E-F). The presence of $\mathrm{i}-\mathrm{NOS}$ revealed by the anti-i-NOS antibodies conjugated to fluorescent FITC was observed in all experimental conditions tested $(B, D$ and F). Bars $=10 \mathrm{~nm}$

antioxidant potential by reducing $\mathrm{Fe}(\mathrm{III})$ to $\mathrm{Fe}(\mathrm{II})$, ensuring the balance of its redox chemical microenvironment and minimising the effect of oxidation of fundamental structures on fungal growth.

The novel findings of this work led us to propose that the melanin of $F$. pedrosoi reacts with ferric iron to reduce it to ferrous iron, and maintains this iron-melanin complex as a redox buffer to trap oxidative radicals. This explains the higher growth rate of the control F. pedrosoi samples compared to the TC-treated samples following exposure to NO and hydrogen peroxide (Fig. 4), as well as the higher susceptibility of the TC-treated samples to activated macrophages [12].

The progressive microwave power saturation ESR experiments, which varied the power of the microwaves on the magnetised sample, showed approximately a two times higher intensity in the control-melanin samples compared to the TC-melanin samples. According to our 
Table 1: Nitrite concentration after fungal interaction with activated murine macrophages.

\begin{tabular}{|c|c|c|}
\hline & \multicolumn{2}{|c|}{ Nitrite concentration $(\mu \mathrm{M})^{*}$} \\
\hline Activated murine macrophages & After $24 \mathrm{~h}$ & After $48 \mathrm{~h}$ \\
\hline Without fungus & $20.0 \pm 0.70$ & $50.0 \pm 0.70$ \\
\hline With F. pedrosoi & $1.9 \pm 0.40$ & $4.0 \pm 0.28$ \\
\hline $\begin{array}{l}\text { With } 1 \mu \mathrm{g} / \mathrm{ml} \text { of melanin isolated from F. pe- } \\
\text { drosoi }\end{array}$ & $0.9 \pm 0.54$ & $1.1 \pm 0.14$ \\
\hline With TC-treated F. pedrosoi & $36.2 \pm 1.25$ & $50.0 \pm 3.95$ \\
\hline
\end{tabular}

hypothesis, this suggests that control-melanin has more self-interaction sites as well as interaction sites for associated structures and therefore is more compact. As indicated by Herbst et al. [29], the profile of progressive microwave power saturation curves of amorphous solids

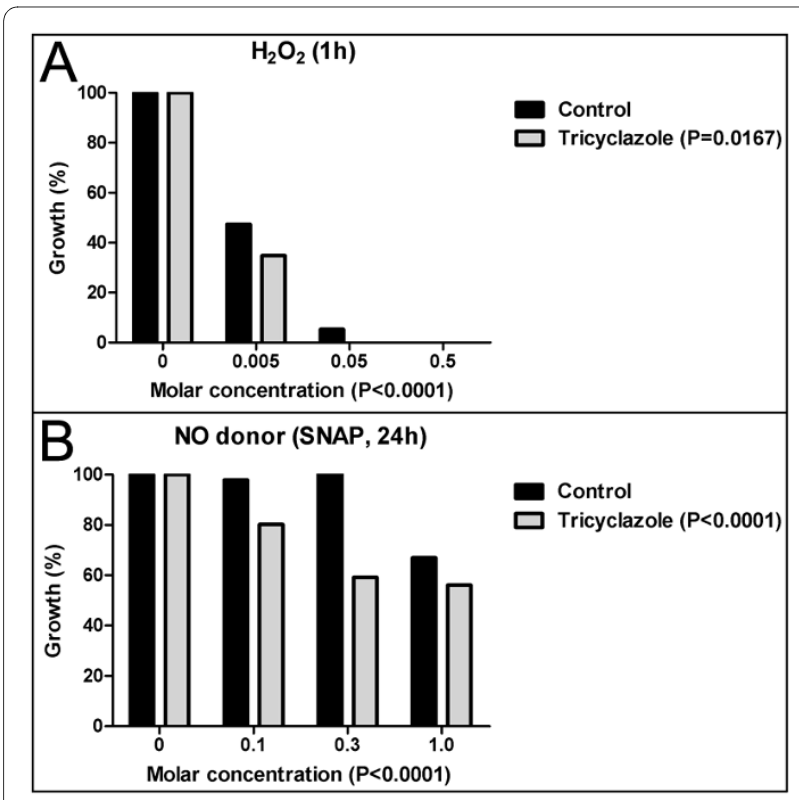

Figure 4 Fungal growth after exposure to $\mathrm{H}_{2} \mathrm{O}_{2}$ and NO. Graphic representation of the growth of $F$. pedrosoi with (gray bars) or without (black bars) tricyclazole (TC) treatment after exposure to $\mathrm{H}_{2} \mathrm{O}_{2}$ for $1 \mathrm{~h}$ (A), or the NO donor SNAP for $24 \mathrm{~h}$ (B). After exposure to $\mathrm{H}_{2} \mathrm{O}_{2}$ or NO, the growth of the TC-treated $F$. pedrosoi was less pronounced than that of the control fungus $(\mathrm{P}<0.05)$. Values are the percentage of growth relative to the control or TC-treated fungi not exposed to $\mathrm{H}_{2} \mathrm{O}_{2}$ or $\mathrm{NO}$. is linked to the effectiveness of spin relaxation pathways for the paramagnetic centre that interacts with its surroundings. Hence, the measure of the progressive microwave power saturation curves for similar paramagnetic centres may provide an indirect indication of molecular arrangements. In this study, the profiles observed for control-melanin (Fig. 1) suggest that it is a more compact polymer than TC-melanin because its spin relaxation rates are faster. Such data are in agreement with the thinner cell wall of untreated $F$. pedrosoi conidia compared to TC-treated $F$. pedrosoi as revealed by freeze-fracture assays [30].

Our data from interaction assays between fungi and activated murine macrophages suggest that melanin is involved in the protection of the fungus against NO. Precipitation of formazan in the NBT assay (Fig. 2) revealed the presence of an oxidative response in the interface between the melanin-free fungi and macrophages. These experiments also showed that the presence of controlmelanin (either free in the media or adhered to the fungal cell) decreased NO levels as revealed by its direct correlation to the detected nitrite levels. Further, TC-treatment of $F$. pedrosoi conidia resulted in at least an $80 \%$ increase in the amount of nitrite detected after the first $24 \mathrm{~h}$ of interaction compared to samples with only macrophages. These data indicate that the inhibition of the melanin pathway, and consequently, the absence of melanin exposed on the cell wall of the fungus, could stimulate the production of NO by activated macrophages.

Fungal glucans, the major component of the fungal cell wall, were previously described to activate macrophages (which express glucan receptors) and promote the syn- 
thesis and release of NO [31]. Nimrichter et al. [32] suggested that the removal of melanin from the $F$. pedrosoi cell wall exposes antigens, such as glucans, that were previously masked by melanin. We conclude that the increase of the macrophages' oxidative response after interaction with TC-treated F. pedrosoi was probably due to the unmasking of antigens/glucans in the fungal cell wall.

The inhibition of i-NOS expression by pathogens has been reported in other microorganisms, e.g., Toxoplasma gondii [33]. Bocca el al. [34] suggested that melanin from F. pedrosoi could inhibit NO production in macrophages. However, our experiments suggest that the reduction of nitrite levels after the interaction of macrophages and control conidia was not due the inhibition of i-NOS expression, since its expression was detected in all tested conditions in immunofluorescence experiments. We propose that $F$. pedrosoi melanin acts as a scavenger of oxidative radicals, masking the detection of $\mathrm{NO}$ in some systems.

The conversion of L-arginine by $\mathrm{i}-\mathrm{NOS}$ in the presence of $\mathrm{NO}$ requires calcium ions and $\mathrm{Fe}(\mathrm{III})$ (in an heme group). Melanin participates in the storage of calcium and iron in F. pedrosoi, and therefore it might reduce the availability of such ions in the interaction microenvironment $[11,35]$. In addition, NO reversibly reacts with both $\mathrm{Fe}(\mathrm{III})$ and $\mathrm{Fe}(\mathrm{II})$, leaving an electron that could remain trapped in the quinone groups of melanin $[8,36]$.

The assays with the NO donor SNAP and $\mathrm{H}_{2} \mathrm{O}_{2}$ revealed that untreated $F$. pedrosoi grew more than TCtreated F. pedrosoi; this suggests a protective function for melanin. In these experiments, our only variables were the F. pedrosoi conidia and the oxidative agent. Consequently, in these systems, no other mechanism can occur to inhibit i-NOS production.

\section{Conclusions}

Our data suggest a protective role for $F$. pedrosoi melanin by its direct interaction with $\mathrm{NO}$; the fungal melanin acts as a trap for the unpaired electron of NO, protecting the fungus against oxidative damage. This mechanism impairs the immune system and makes difficult the fungal clearance by macrophages and other phagocytes, which might be related to the recalcitrant nature of chromoblastomycosis lesions and the chronic nature of this disease. Further investigation is necessary to define the structure of fungal melanins and describe putative chemical reactions that could occur in the infection environment, the products of such reactions and possible target sites for the development of new drugs.

\section{Methods}

\section{Microorganism and reagents}

A human isolate of F. pedrosoi (5VLP) [37] was inoculated in modified Czapek Dox (CD) liquid media (Sucrose $30 \mathrm{~g} /$ $\mathrm{L}, \mathrm{NaNO}_{3} 2 \mathrm{~g} / \mathrm{L}, \mathrm{KH}_{2} \mathrm{PO}_{4} 1 \mathrm{~g} / \mathrm{L}, \mathrm{MgSO}_{4} \cdot 7 \mathrm{H}_{2} \mathrm{O} 0.5 \mathrm{~g} / \mathrm{L}$, $\mathrm{KCl} 0.5 \mathrm{~g} / \mathrm{L}$, ammoniacal iron citrate $0.01 \mathrm{~g} / \mathrm{L}$ ), $\mathrm{pH} 5.5$, with shaking at $28^{\circ} \mathrm{C}$ for five days. TC (kindly provided by Dow AgroSciences, Indianapolis, USA) was dissolved in dimethylsulphoxide (DMSO) and added to cultures at a final concentration of $16 \mu \mathrm{g} / \mathrm{ml}$ to block the DHN-melanin biosynthesis pathway. All other reagents were acquired from Sigma-Aldrich (Brazil), unless otherwise specified. Saccharomyces cerevisiae (INCQS 40001, ATCC 2601) was donated by Coleção de Culturas de Fungos of Instituto Oswaldo Cruz, Rio de Janeiro, Brazil.

\section{Melanin isolation}

F. pedrosoi melanins were isolated from fungal cultures following incubation with $16 \mu \mathrm{g} / \mathrm{ml}$ of TC (TC-melanin) or without the drug (control-melanin) by an alkali-acid extraction method described elsewhere [6].

\section{Electron Spin Resonance}

After isolation, melanins $(10 \mathrm{mg})$ from $F$. pedrosoi cultures were thoroughly triturated manually in a solid marble mortar with a pestle. The trituration was a necessary step in order to diminish the grain size, which otherwise could lead to preferential orientations and to the observation of artifacts in the ESR spectra. The pigments were analysed by ESR spectroscopy coupled to a spin-trapping analysis. The spectra were acquired at room temperature in quartz tubes on a Bruker ESP 380-E CW/FT spectrometer (Bruker, Germany) operating at X-Band $(9.5 \mathrm{GHz})$. The amplitude modulation was kept constant at 3.0 gauss and low power microwaves were used to avoid saturation. The microwave power saturation experiments were measured between 0.02-200 $\mathrm{mW}$, while all others parameters remained the same. The $g$ factors (the ESR quantity analogous to the chemical shift in nuclear magnetic ressonance spectroscopy), which are related to the magnetic field, were measured upon a diphenylpicrylhydrazyl radi$\mathrm{cal}(\mathrm{DPPH})$ standard, $\mathrm{g}=2.0023$ [38].

\section{Conidia Isolation}

F. pedrosoi cells with or without a treatment of $16 \mu \mathrm{g} / \mathrm{ml}$ of TC were filtered in a 40-60G porous plate filter, followed by conidia recovery by centrifugation $(13,600 \mathrm{~g}, 30$ $\min , 4^{\circ} \mathrm{C}$ ).

\section{Peritoneal Macrophages}

Peritoneal washes with Hanks' Balanced Salt Solution were performed in 2-3-week-old Swiss male mice. Resident macrophages were seeded on glass coverslips in 24well plates or in Petri dishes for $1 \mathrm{~h}$ at $37^{\circ} \mathrm{C}$ in a $5 \% \mathrm{CO}_{2}$ 
atmosphere. Cells were then washed and cultured for 24 $\mathrm{h}$ in DMEM containing 10\% foetal bovine serum.

\section{Macrophage Activation and Fungal-Host Cell Interaction}

Macrophages were activated with $50 \mathrm{U} / \mathrm{ml}$ of recombinant murine IFN- $\gamma$ and $100 \mathrm{ng} / \mathrm{ml}$ of LPS from Escherichia coli 0111:B4 for $24 \mathrm{~h}$ before interaction with the fungus. Interactions were carried out at a 10:1 (fungus:macrophage) ratio for $24 \mathrm{~h}$ at $37^{\circ} \mathrm{C}$ in a $5 \% \mathrm{CO}_{2}$ atmosphere.

\section{Oxidative Burst}

Conidia were extracted from cultures of $F$. pedrosoi grown in three different conditions: (I) aeration with exposure to light; (II) low aeration in the dark; (III) and supplemented with $16 \mu \mathrm{g} / \mathrm{ml}$ of TC. S. cerevisiae was also used in two different conditions: (I) alone as a control or (II) supplemented with $1 \mu \mathrm{g} / \mathrm{ml}$ of melanin isolated from F. pedrosoi. The interaction of fungal cells with activated murine macrophages was evaluated on round glass coverslips in 24-well plates using DMEM defined medium supplemented with $0.5 \mathrm{mg} / \mathrm{ml}$ of nitroblue tetrazolium (NBT; grade 111), for $15 \mathrm{~min}$ at $37^{\circ} \mathrm{C}$. After this incubation, nonadherent and non-internalised fungal cells were removed by gentle washes with PBS. The coverslips were again incubated in DMEM for 30 min to reduce background signals, fixed using Bouin's solution, dehydrated in acetone-xylol and mounted in Entellan resin. The oxidative response of the samples was scored as positive after the observation of the precipitation of indigo blue (formazan) around fungal cells in randomly chosen fields under a bright field light microscope.

\section{Nitrite evaluation}

NO detection was evaluated indirectly by measuring the nitrite levels in macrophage cultures supernatants after interaction as described elsewhere [39]. Briefly, macrophages and fungi (at a fungus to macrophage ratio of 10:1) were allowed to interact for 24 or $48 \mathrm{~h}$ in DMEM at $37^{\circ} \mathrm{C}, 5 \% \mathrm{CO}_{2}$. Macrophages culture conditions were the following: (I) macrophages cultured alone; (II) macrophages with TC-treated conidia; (III) macrophages with control F. pedrosoi; and (IV) macrophages cultured with $1 \mu \mathrm{g} / \mathrm{ml}$ of melanin extracted from $F$. pedrosoi. Supernatant from each well $(100 \mu \mathrm{l})$ was mixed with an equal volume of Griess reagent in a 96-well flat-bottomed plate. The absorbance at $540 \mathrm{~nm}$ was measured with a Dynatech MR 5000 Microplate Reader. The nitrite concentration was calculated from a standard curve of sodium nitrite diluted in DMEM.

\section{i-NOS expression detected by immunofluorescence}

Macrophages before or after interaction with $F$. pedrosoi conidia with or without TC treatment were fixed for 30 min in 3\% formaldehyde in PBS. These samples were incubated for $20 \mathrm{~min}$ in $50 \mathrm{mM}$ ammonium chloride in PBS and then washed for $10 \mathrm{~min}$ in PBS with bovine serum albumin (PBS-BSA). Cells were then incubated for 40 min with rabbit polyclonal antibody for mouse i-NOS (Santa Cruz Biotechnology, CA, USA) diluted 1:100 in PBS-BSA. Cells were washed twice with PBS-BSA and incubated for $30 \mathrm{~min}$ with a FITC-labelled goat anti-rabbit IgG diluted 1:200 in PBS-BSA. The cells were then mounted with N-propyl gallate and observed and photographed in a Zeiss confocal laser scanning microscope.

\section{Fungal growth after treatment with hydrogen peroxide}

$\mathrm{H}_{2} \mathrm{O}_{2}$ (Merck, USA) was added directly to control and TC-treated cultures to final concentrations of $0.005,0.05$ and $0.5 \mathrm{M}$. Conidia $\left(2 \times 10^{3}\right.$ cells $\left./ \mathrm{ml}\right)$ were incubated in RPMI-1640, for $1 \mathrm{~h}$ at $37^{\circ} \mathrm{C}$ in the presence of the hydrogen peroxide concentrations mentioned above. From each sample, $50 \mu \mathrm{l}$ were placed in wells of a 24 -well plate with $500 \mu \mathrm{l}$ of $\mathrm{CD}$ with $3 \%$ agar. The cultures were incubated at $25^{\circ} \mathrm{C}$ for 10 days.

Fungal growth was measured by calculating the relative size of the colonies per well for each condition. Images of the bottom of the plates were digitalised and processed using ImageJ software [40] for the following parameters: (I) gamma correction to ensure adequate brightness and contrast of the image; (II) a threshold to define the interface between the fungal growth (black) and the background (white); and for (III) the inversion to define the background as black (grayscale value $=0$ ) and the area of fungal growth as white (grayscale value $=255$ ). A constant area with the diameter of a well from a 24-well plate was the template for the measurements of the "Mean Gray Value" on the Image J software. Measurements were the sum of the gray values of all pixels in the selection divided by the number of pixels, revealing the area of fungal growth. In this work the values were expressed as the normalised percentage relative to its control $(100 \%$ of growth).

\section{Fungal growth after incubation with a nitric oxide donor}

SNAP, a nitric oxide donor, was dissolved in DMSO and added to untreated and TC-treated cultures of conidia (2 $\times 10^{3}$ cells $/ \mathrm{ml}$ ) in RPMI- 1640 at concentrations of $0.1,0.3$ and $1.0 \mathrm{mM}$. These cultures were incubated for $24 \mathrm{~h}$ at $37^{\circ} \mathrm{C}$. From each condition, $50 \mu \mathrm{l}$ were plated in one well of a 24-well plate with $500 \mu \mathrm{l}$ of CD (solid, with $3 \%$ agar). Samples were incubated at $25^{\circ} \mathrm{C}$ for 10 days. The growth area was measured and using the procedure described above.

\section{Statistical analysis}

Graphic and statistical analyses were performed with GraphPad Prism 5.0 (GraphPad Software, USA). The Student's $t$-test was used for experiments with one variable, 
and results were considered significant if $\mathrm{P}<0.0001$. ANOVA tests were used for comparing samples in experiments with more than one variable; the results were considered significant when $\mathrm{P}<0.05$.

\section{Authors' contributions}

MMLC, AJF, SHS, WS and SR conceived of the study and participated in its design and the writing of this paper. MMLC, AJF and SHS performed the experiments with murine macrophages. MMLC and LPB performed the experiments investigating the activity of oxidative species. MMLC, MHH and NVV performed the ESR experiments. All authors read and approved the final manuscript.

\section{Acknowledgements}

This work was supported by grants from Conselho Nacional de Desenvolvimento Científico e Tecnológico (CNPq) and Fundação Carlos Chagas Filho de Amparo à Pesquisa do Estado do Rio de Janeiro (FAPERJ).

\section{Author Details}

IInstituto de Biofísica Carlos Chagas Filho (IBCCF), Centro de Ciências da Saúde (CCS), Universidade Federal do Rio de Janeiro (UFRJ), Rio de Janeiro, RJ, Brazil, 2Laboratório de Tecnologia em Bioquímica e Microscopia, Centro Universitário da Zona Oeste (UEZO), Rio de Janeiro, Brazil, 3Instituto de Ciências Exatas, Departamento de Química, Universidade Federal Rural do Rio de Janeiro, RJ, Brazil and 4 Instituto de Física, Universidade Federal do Rio de Janeiro, RJ, Brazil

Received: 2 September 2009 Accepted: 16 March 2010

Published: 16 March 2010

\section{References}

1. Lopez Martinez R, Mendez Tovar LJ: Chromoblastomycosis. Clin Dermatol 2007, 25:188-194.

2. Silva JP, de Souza W, Rozental S: Chromoblastomycosis: a retrospective study of 325 cases on Amazonic Region (Brazil). Mycopathologia 1998, 143:171-175.

3. Salgado CG, da Silva JP, da Silva MB, da Costa PF, Salgado UI: Cutaneous diffuse chromoblastomycosis. Lancet Infect Dis 2005, 5:528.

4. Queiroz-Telles F, Esterre P, Perez-Blanco M, Vitale RG, Salgado CG, Bonifaz A: Chromoblastomycosis: an overview of clinical manifestations, diagnosis and treatment. Med Mycol 2009, 47:3-15.

5. Santos AL, Palmeira VF, Rozental S, Kneipp LF, Nimrichter L, Alviano DS, Rodrigues ML, Alviano CS: Biology and pathogenesis of Fonsecaea pedrosoi, the major etiologic agent of chromoblastomycosis. FEMS Microbiol Rev 2007, 31:570-591.

6. Alviano CS, Farbiarz SR, De Souza W, Angluster J, Travassos LR: Characterization of Fonsecaea pedrosoi melanin. J Gen Microbiol 1991, 137:837-844.

7. Langfelder K, Streibel M, Jahn B, Haase G, Brakhage AA: Biosynthesis of fungal melanins and their importance for human pathogenic fungi. Fungal Genet Biol 2003, 38:143-158.

8. Jacobson ES: Pathogenic roles for fungal melanins. Clin Microbiol Rev 2000, 13:708-717.

9. Nosanchuk JD, Casadevall A: The contribution of melanin to microbial pathogenesis. Cell Microbiol 2003, 5:203-223.

10. Franzen AJ, de Souza W, Farina M, Alviano CS, Rozental S: Morphometric and densitometric study of the biogenesis of electron-dense granules in Fonsecaea pedrosoi. FEMS Microbiol Lett 1999, 173:395-402

11. Franzen AJ, Cunha MM, Miranda K, Hentschel J, Plattner H, da Silva MB, Salgado CG, de Souza W, Rozental S: Ultrastructural characterization of melanosomes of the human pathogenic fungus Fonsecaea pedrosoi. $J$ Struct Biol 2008, 162:75-84

12. Cunha MM, Franzen AJ, Alviano DS, Zanardi E, Alviano CS, De Souza W, Rozental S: Inhibition of melanin synthesis pathway by tricyclazole increases susceptibility of Fonsecaea pedrosoi against mouse macrophages. Microsc Res Tech 2005, 68:377-384.

13. Farbiarz SR, de Carvalho TU, Alviano C, de Souza W: Inhibitory effect of melanin on the interaction of Fonsecaea pedrosoi with mammalian cells in vitro. J Med Vet Mycol 1992, 30:265-273.

14. Sotto MN, De Brito T, Silva AM, Vidal M, Castro LG: Antigen distribution and antigen-presenting cells in skin biopsies of human chromoblastomycosis. J Cutan Pathol 2004, 31:14-18.
15. Lane TE, Otero GC, Wu-Hsieh BA, Howard DH: Expression of inducible nitric oxide synthase by stimulated macrophages correlates with their antihistoplasma activity. Infect Immun 1994, 62:1478-1479.

16. Rossi GR, Cervi LA, Garcia MM, Chiapello LS, Sastre DA, Masih DT: Involvement of nitric oxide in protecting mechanism during experimental cryptococcosis. Clin Immunol 1999, 90:256-265.

17. Fernandes KS, Coelho AL, Lopes Bezerra LM, Barja-Fidalgo C: Virulence of Sporothrix schenckii conidia and yeast cells, and their susceptibility to nitric oxide. Immunology 2000, 101:563-569.

18. Hamilton AJ, Holdom MD: Antioxidant systems in the pathogenic fungi of man and their role in virulence. Med Mycol 1999, 37:375-389.

19. de Cassia RGR, Pombeiro-Sponchiado SR: Antioxidant activity of the melanin pigment extracted from Aspergillus nidulans. Biol Pharm Bull 2005, 28:1129-1131.

20. Nosanchuk JD, Casadevall A: Impact of melanin on microbial virulence and clinical resistance to antimicrobial compounds. Antimicrob Agents Chemother 2006, 50:3519-3528.

21. Wang Y, Aisen P, Casadevall A: Melanin, melanin "ghosts," and melanin composition in Cryptococcus neoformans. Infect Immun 1996, 64:2420-2424

22. Nosanchuk JD, van Duin D, Mandal P, Aisen P, Legendre AM, Casadevall A: Blastomyces dermatitidis produces melanin in vitro and during infection. FEMS Microbiol Lett 2004, 239:187-193.

23. Gomez BL, Nosanchuk JD, Diez S, Youngchim S, Aisen P, Cano LE, Restrepo A, Casadevall A, Hamilton AJ: Detection of melanin-like pigments in the dimorphic fungal pathogen Paracoccidioides brasiliensis in vitro and during infection. Infect Immun 2001, 69:5760-5767.

24. Nosanchuk JD, Gomez BL, Youngchim S, Diez S, Aisen P, Zancope-Oliveira RM, Restrepo A, Casadevall A, Hamilton AJ: Histoplasma capsulatum synthesizes melanin-like pigments in vitro and during mammalian infection. Infect Immun 2002, 70:5124-5131.

25. Morris-Jones R, Youngchim S, Gomez BL, Aisen P, Hay RJ, Nosanchuk JD, Casadevall A, Hamilton AJ: Synthesis of melanin-like pigments by Sporothrix schenckii in vitro and during mammalian infection. Infect Immun 2003, 71:4026-4033.

26. Paolo WF Jr, Dadachova E, Mandal P, Casadevall A, Szaniszlo PJ, Nosanchuk JD: Effects of disrupting the polyketide synthase gene WdPKS1 in Wangiella [Exophiala] dermatitidis on melanin production and resistance to killing by antifungal compounds, enzymatic degradation, and extremes in temperature. BMC Microbiol 2006, 6:55.

27. Krzywda A, Petelenz E, Michalczyk D, Plonka PM: Sclerotia of the acellular (true) slime mould Fuligo septica as a model to study melanization and anabiosis. Cell Mol Biol Lett 2008, 13:130-143.

28. Jacobson ES, Hong JD: Redox buffering by melanin and Fe(II) in Cryptococcus neoformans. J Bacteriol 1997, 179:5340-5346.

29. Herbst MH, Pinhal NM, Demétrio FAT, Dias GHM, Vugman NV: Solid-state structural studies on amorphous platinum-fullerene[60] compounds [PtnC60] $(n=1,2)$. Journal of Non-Crystalline Solids 2000, 272:127-130.

30. Franzen AJ, Cunha MM, Batista EJ, Seabra SH, De Souza W, Rozental S: Effects of tricyclazole (5-methyl-1,2,4-triazol[3,4] benzothiazole), a specific DHN-melanin inhibitor, on the morphology of Fonsecaea pedrosoi conidia and sclerotic cells. Microsc Res Tech 2006, 69:729-737.

31. Kataoka K, Muta T, Yamazaki S, Takeshige K: Activation of macrophages by linear (1 right-arrow3)-beta-D-glucans. Impliations for the recognition of fungi by innate immunity. J Biol Chem 2002, 277:36825-36831

32. Nimrichter L, Cerqueira MD, Leitao EA, Miranda K, Nakayasu ES, Almeida SR, Almeida IC, Alviano CS, Barreto-Bergter E, Rodrigues ML: Structure, cellular distribution, antigenicity, and biological functions of Fonsecaea pedrosoi ceramide monohexosides. Infect Immun 2005, 73:7860-7868.

33. Rozenfeld C, Martinez R, Seabra S, Sant'anna C, Goncalves JG, Bozza M, Moura-Neto V, De Souza W: Toxoplasma gondii prevents neuron degeneration by interferon-gamma-activated microglia in a mechanism involving inhibition of inducible nitric oxide synthase and transforming growth factor-beta1 production by infected microglia. Am J Pathol 2005, 167:1021-1031.

34. Bocca AL, Brito PP, Figueiredo F, Tosta CE: Inhibition of nitric oxide production by macrophages in chromoblastomycosis: a role for Fonsecaea pedrosoi melanin. Mycopathologia 2006, 161:195-203.

35. Alderton WK, Cooper CE, Knowles RG: Nitric oxide synthases: structure, function and inhibition. Biochem J 2001, 357:593-615. 
36. Gutteridge JM, Halliwell B: Free radicals and antioxidants in the year 2000. A historical look to the future. Ann N Y Acad Sci 2000, 899:136-147.

37. Oliveira LG, Resende MA, Lopes CF, Cisalpino EO: Isolamento e identificação dos agentes da cromomicose em Belo Horizonte. Rev Soc Bras Med Trop 1973, 7:1.

38. Weil JA, Bolton JR: Electron Paramagnetic Resonance: Elementary Theory and Practical Applications 2nd edition. 1972.

39. Green LC, Wagner DA, Glogowski J, Skipper PL, Wishnok JS, Tannenbaum SR: Analysis of nitrate, nitrite, and [15N]nitrate in biological fluids. Anal Biochem 1982, 126:131-138.

40. Rasband WS: ImageJ. Bethesda, Maryland: National Institutes of Health; 1997.

doi: $10.1186 / 1471-2180-10-80$

Cite this article as: Cunha et al., Melanin in Fonsecaea pedrosoi: a trap for oxidative radicals BMC Microbiology 2010, 10:80

Submit your next manuscript to BioMed Central and take full advantage of:

- Convenient online submission

- Thorough peer review

- No space constraints or color figure charges

- Immediate publication on acceptance

- Inclusion in PubMed, CAS, Scopus and Google Scholar

- Research which is freely available for redistribution

Submit your manuscript at www.biomedcentral.com/submit 